\title{
SSR Molecular Markers Development Based on Whole Genome Sequences in
} Glycyrrhiza uralensis Fisch.

Zhan Haixian, Wang Yingli, Du Chenhui, Zhang Dan, Li Rui, Yang Mengru, Zhang Shuosheng $\unrhd$

School of Chinese Materia Medica and Food Engineering, Shanxi University of Chinese Medicine, Jinzhong, 030619, China

$\nabla$ Corresponding author email: zhangshuosheng@aliyun.com

Medicinal Plant Research, 2020, Vol.10, No.4 doi: 10.5376/mpr.2020.10.0004

Received: 26 May., 2020

Accepted: 11 Jun., 2020

Published: 30 Jun., 2020

Copyright $(\mathbf{2 0 2 0}$ Zhan et al., This article was first published in Molecular Plant Breeding in Chinese, and here was authorized to translate and publish the paper in English under the terms of Creative Commons Attribution License, which permits unrestricted use, distribution, and reproduction in any medium, provided the original work is properly cited.

Preferred citation for this article:

Zhan H.X., Wang Y.L., Du C.H., Zhang D., Li R., Yang M.R., and Zhang S.S., 2020, SSR molecular markers development based on whole genome sequences in Glycyrrhiza uralensis Fisch., Medicinal Plant Research, 10(4): 1-8 (doi: 10.5376/mpr.2020.10.0004)

\begin{abstract}
It is different in terms of active ingredients from various sources and origins on Glycyrrhiza Linn.. The development of specific molecular markers is of great significance for identification of germplasm resources. In the study, simple sequence repeat (SSR) motifs were analyzed based on the whole genome data in Glycyrrhiza uralensis Fisch.. SSRs were identified with MISA tool in all scaffold sequences. Suitable SSRs were used to design primers by using Primer3-2.4.0 software and 100 pairs of SSR primers were randomly utilized for the validity of molecular markers. The results showed 193207 SSRs were detected from the whole genome sequence of $G$. uralensis distributed in 9250 scaffolds. Of these, mono-nucleotide repeat SSRs was the main type, accounting for $60.73 \%$, followed by dinucleotide $(26.11 \%)$ and trinucleotide repeat $(10.95 \%)$. Of the 284 repeat motifs, A/T (58.28\%), AG/CT (10.48\%), AT/AT (10.48\%), AC/GT (5.12\%) and AAT/ATT (3.57\%) were the main repeat base. In addition, SSR primers for 140294 SSRs were designed using Primer3-2.4.0 software. We eventually developed 701302 pairs of SSR markers in $G$. uralensis. Subsequently, 100 pairs of SSR primers were randomly selected for the further verifying. PCR results indicated that 63 of them (63\%) had amplified the target bands, and 12 pairs of primers could generate polymorphic bands in seven samples. A large number of SSR markers developed will be used for identification of Glycyrrhiza germplasm resources and future molecular marker assisted breeding.
\end{abstract}

Keywords Glycyrrhiza uralensis Fisch.; Genome; Repetitive sequence; SSR marker; Development

Glycyrrhiza uralensis Fisch. is a perennial herb or subshrub of Glycyrrhiza in Leguminosae. Its roots and rhizomes have the effects of moistening lung and relieving cough, tonifying spleen and supporting Qi, clearing away heat and relieving toxin, harmonizing drugs and so on, which is one of the traditional Chinese medicines (Chinese Pharmacopoeia Commission, 2015). In addition to its medicinal value, Glycyrrhiza uralensis Fisch. also has the functions of wind prevention, sand fixation, and water and soil conservation. In recent years, with the application of Glycyrrhiza uralensis Fisch. in agriculture, food, cosmetics, industry and other fields, its demand has also increased accordingly. Due to over excavation, the distribution area of wild Glycyrrhiza uralensis Fisch. has been decreasing year by year, and even facing the depletion of wild resources, which has led to the continuous increase of the planting area of cultivated Glycyrrhiza uralensis Fisch.. Large-area planting bases for cultivated Glycyrrhiza uralensis Fisch. have been established in Inner Mongolia, Xinjiang, Jilin and Shanxi respectively (Li, 1993). Studies have shown that even the medicinal ingredients of Glycyrrhiza uralensis Fisch. with the same origin are different (Ruo et al., 2018). In order to better identify species and distinguish germplasm resources, it is particularly important to develop molecular markers associated with specific traits of Glycyrrhiza uralensis Fisch..

Molecular markers have been widely used in genetic identification and genetic background analysis of medicinal plants. The development of molecular markers of Glycyrrhiza uralensis Fisch. mainly involves random amplified polymorphic DNA (RAPD), amplified fragment length polymorphism (AFLP) (Liu et al., 2015), inter-simple sequence repeat (ISSR) (Li et al., 2010), start codon targeted polymorphism (SCoT), expression sequence tag simple sequence repeat (EST-SSR) (Li et al., 2015) and simple sequence repeat (SSR) markers (Liu et al., 2015). Among them, SSR markers are DNA fragments composed of 1 6 bp nucleotides, which are widely distributed in the genomes of eukaryotes. An SSR sequence appears at an average of about $10 \mathrm{~kb}$ nucleotides, and there are 
highly conserved sequences at both ends. Due to the different SSR sequences and repetition times of different species, SSR markers show a high degree of conservatism and repeatability in different species, so that different species can be accurately identified. At present, most of the published SSR markers of Glycyrrhiza uralensis Fisch. were developed based on the sequencing results of Glycyrrhiza uralensis Fisch. transcriptome. But there were such limited molecular markers of Glycyrrhiza uralensis Fisch. that could not meet the needs of identification of germplasm resources and genetic diversity analysis (Liu et al., 2015). In this study, based on the whole genome sequences in Glycyrrhiza uralensis Fisch., the composition and frequency of SSRs in Glycyrrhiza uralensis Fisch. genome were statistically analyzed by bioinformatics methods, and a large number of SSR markers were designed. Seven Glycyrrhiza uralensis Fisch. samples were used to verify the designed markers. The developed polymorphic markers can be used for the identification of germplasm resources, genetic diversity analysis and molecular marker assisted breeding, which could lay a foundation for artificially breeding excellent Glycyrrhiza uralensis Fisch. varieties.

\section{Results and Analysis}

\subsection{Distribution of SSRs in Glycyrrhiza uralensis Fisch. genome}

Using MISA to search for repeat loci based on 12528 scaffolds in the whole genome of Glycyrrhiza uralensis Fisch., it was found that 9250 scaffolds contained SSR loci, the frequency was $73.52 \%$, and the number of SSRs was 193 207, including 32112 complex microsatellites. There were 510 SSR loci in each Mb sequence of the genome, and on average, each scaffold contained 179 SSR loci. 1 6 nucleotide positions were all present, and the number of mononucleotide, dinucleotide, trinucleotide, tetranucleotide, pentanucleotide, and hexanucleotide repeat units were $117343,50445,21159,2889,844$ and 527, which respectively accounted for $60.73 \%, 26.11 \%$, $10.95 \%, 1.50 \%, 0.44 \%$ and $0.27 \%$ of the total number of repeat units (Table 1 ; Figure $1 \mathrm{~A}$ ).

Table 1 Number and distribution of SSR loci in Glycyrrhiza uralensis Fisch. genome

\begin{tabular}{|c|c|c|c|c|c|c|c|c|c|c|c|}
\hline \multirow[t]{2}{*}{ Repeat types } & \multicolumn{9}{|c|}{$\underline{\text { Repeat times }}$} & \multirow[t]{2}{*}{ Total } & \multirow{2}{*}{$\begin{array}{l}\text { Percentage } \\
(\%)\end{array}$} \\
\hline & 5 & 6 & 7 & 8 & 9 & 10 & 11 & 12 & $>12$ & & \\
\hline Mononucleotide & & & & & & 64634 & 26849 & 12780 & 13080 & 117343 & 60.73 \\
\hline Dinucleotide & & 15663 & 10074 & 7569 & 5660 & 3594 & 2558 & 1706 & 3621 & 50445 & 26.11 \\
\hline Trinucleotide & 9779 & 4595 & 2532 & 1440 & 748 & 520 & 338 & 242 & 965 & 21159 & 10.95 \\
\hline Tetranucleotide & 1623 & 638 & 288 & 134 & 47 & 50 & 32 & 23 & 54 & 2889 & 1.50 \\
\hline Pentanucleotide & 632 & 141 & 44 & 11 & 4 & 5 & 2 & & 5 & 844 & 0.44 \\
\hline Hexanucleotide & 352 & 107 & 39 & 14 & 2 & 10 & 2 & & 1 & 527 & 0.27 \\
\hline Total & 12386 & 21144 & 12977 & 9168 & 6461 & 68813 & 29781 & 14751 & 17726 & 193207 & 100 \\
\hline
\end{tabular}

1.2 Repeat type and frequency of SSR in Glycyrrhiza uralensis Fisch. genome

Through the analysis of the repeat loci, it was found that among the mononucleotide repeat units, the frequency of $\mathrm{A} / \mathrm{T}$ repeat sequences was 112 592, accounting for $95.95 \%$, and the frequency of G/C type was 4751 , accounting for $4.05 \%$ (Figure 1B). There were 4 types of dinucleotide repeat units. Among them, AG/CT and AT/AT repeat sequences have a high frequency, which were 20251 and 20247 respectively, accounting for $80.28 \%$ of dinucleotide repeat units, followed by AC/GT, and the least was CG/CG type, accounting for only $0.12 \%$ (Figure 1C). There were 10 types of trinucleotide repeat units, of which AAT/ATT has the highest frequency, accounting for $32.58 \%$. The frequencies of AAC/GTT, AAG/CTT and ACC/GGT repeat units accounted for $13.72 \%, 17.56 \%$ and $18.56 \%$ respectively. And the frequencies of the other six types were lower, all lower than 10\% (Figure 1D). There were 30 types of tetranucleotide repeat units, of which AAAT/ATTT has a high frequency, accounting for $32.36 \%$. The frequencies of AAAG/CTTT, AATT/AATT, ACAT/ATGT and AGAT/ATCT accounted for 10.56\%, $10.76 \%, 10.52 \%$ and $10.07 \%$ respectively. The frequencies of the other 25 types were lower than $10 \%$ (Figure 1E). There are 78 types of pentanucleotide repeat units, of which AATAT/ATATT has the highest frequency, accounting for $11.61 \%$, and the frequency of the other types was lower than $10 \%$ (Figure $1 \mathrm{~F}$ ). There are 157 types of hexanucleotide repeat units, and the frequency was generally low. Among them, the frequencies of AGAGGG/CCCTCT, AAGAGG/CCTCTT and AAAAAT/ATTTTT were relatively higher, accounting for 3.42\%, $3.61 \%$ and $2.66 \%$ respectively (Figure $1 \mathrm{G}$ ). 


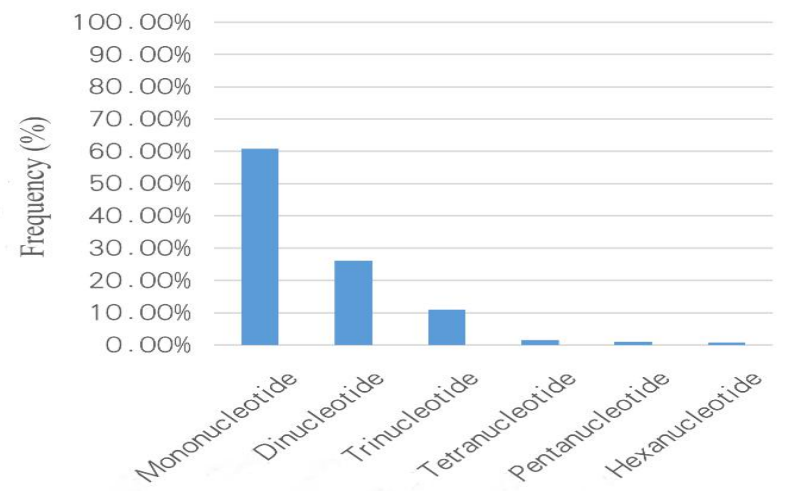

C
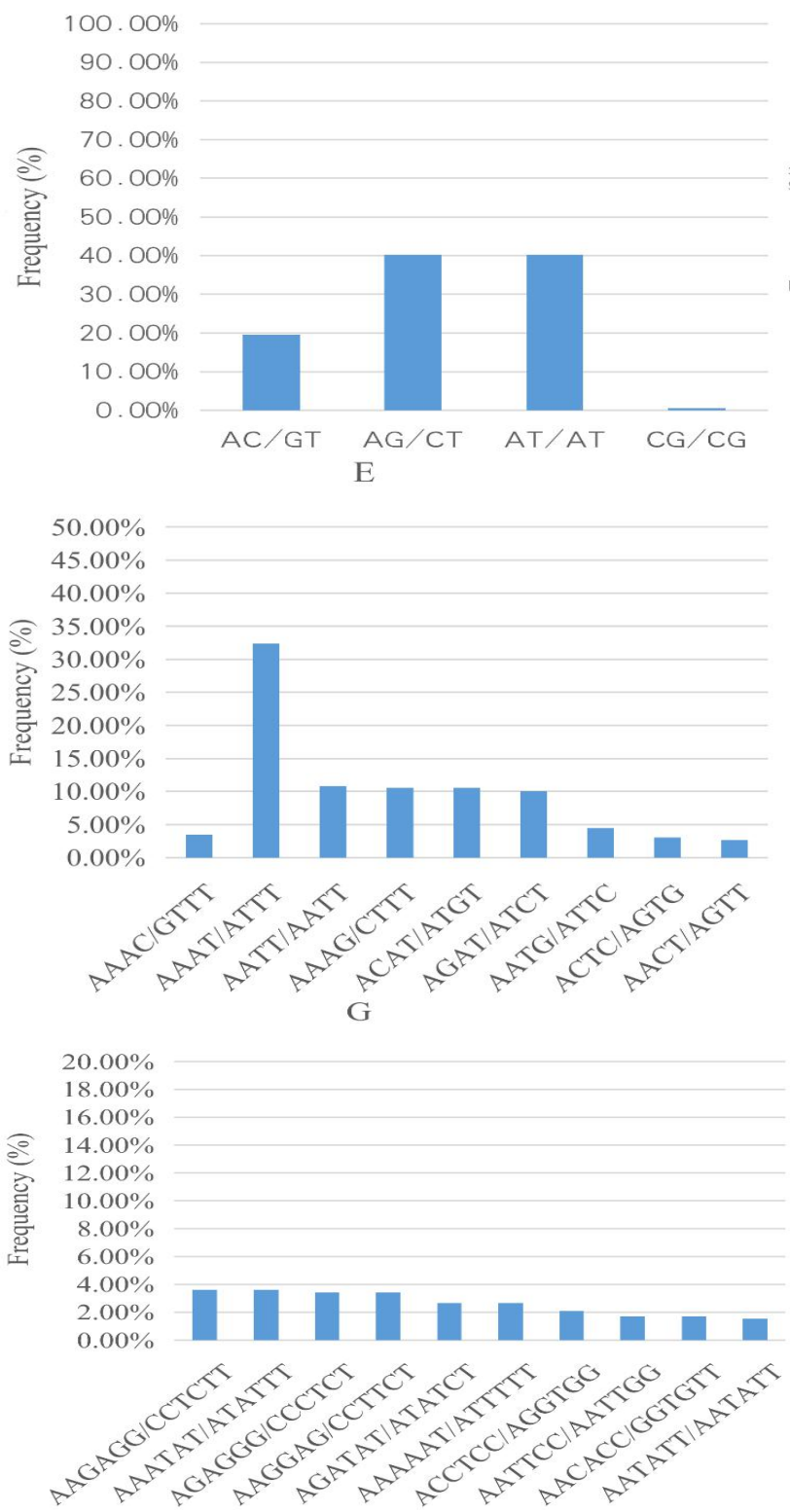

B
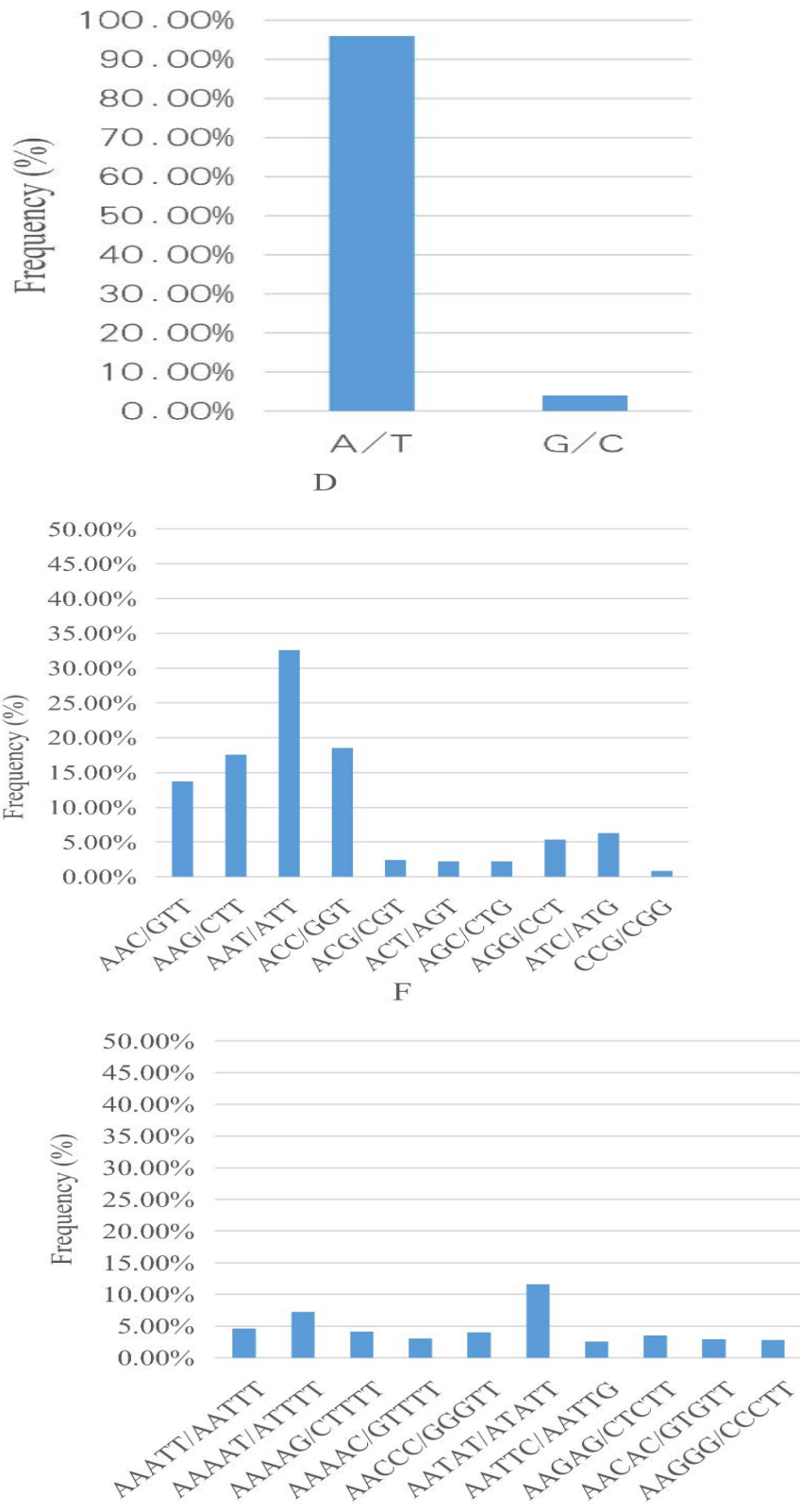

Figure 1 Type and frequency distribution of SSRs

Note: A: Distribution frequency of 6 types of SSR; B: Repeat types and frequency of mononucleotide; C: Repeat types and frequency of dinucleotide; D: Repeat types and frequency of trinucleotide; E: Repeat types and frequency of tetranucleotide; F: Repeat types and frequency of pentanucleotide; G: Repeat types and frequency of hexanucleotide 


\subsection{Design and verification of SSR primers}

140294 repeat loci were selected from all SSRs for primer development, 5 pairs of primers could be designed for most loci, and a total of 701302 pairs of SSR markers were designed (Table 2). 100 pairs of primers designed for scaffolds sequences were randomly selected for marker validity detection. 63 pairs of primers amplified the target bands in seven wild or cultivated Glycyrrhiza uralensis Fisch. from Yanchi County in Xinjiang, Longxi County in Gansu, Shizuishan City in Ningxia, Datong City, Yuci City and Yangqu County in Shanxi, indicating that the effectiveness of primer design was $63 \%$. Among them, 12 pairs of primers could generate polymorphic bands in seven samples, which could be used to identify Glycyrrhiza uralensis Fisch. from different habitats (Figure 2).

Table 2 Information of 12 primer pairs

\begin{tabular}{|c|c|c|c|c|}
\hline Primer No. & Primer sequence (5'-3') & Repeat unit & Annealing temperature $\left({ }^{\circ} \mathrm{C}\right)$ & Expected range (bp) \\
\hline \multirow[t]{2}{*}{ Gcs1 } & F:TCCTGTTTGACCGAACCAACTT & $(\mathrm{TTAA}) 5$ & 61 & 183 \\
\hline & R:TGCACGCAGAGAAGGGAATGAA & & & \\
\hline \multirow[t]{2}{*}{ Gcs2 } & F:TCCTGTTTGACCGAACCAACTT & (A) 11 & 61 & 238 \\
\hline & R:TGCACGCAGAGAAGGGAATGAA & & & \\
\hline \multirow[t]{2}{*}{ Ges3 } & F: GGGAGTGAAGGAGAAGCGAAGA & (TGTGGC)5 & 63 & 209 \\
\hline & R: GGGTCGAGAGGAGAGGGAGAGA & & & \\
\hline \multirow[t]{2}{*}{ Gcs 4} & F: AGGTGTTGGGCATGCGTTTCAG & (TTA) 9 & 59 & 216 \\
\hline & R: GCAGAAGTAGCCAGCTTGATTT & & & \\
\hline \multirow[t]{2}{*}{ Ges5 } & F: CACAACGCAACGCAACCCAAC & $(\mathrm{AACAA}) 5$ & 60 & 250 \\
\hline & R:GGCCTTGGGAGAAGAAGAAGTA & & & \\
\hline \multirow[t]{2}{*}{ Gcs6 } & F: CGTTCTGGTTTTGGTCCTATGG & (AT) 11 & 58 & 248 \\
\hline & R: CCTATGGTCAACTGGTGCTTAA & & & \\
\hline \multirow[t]{2}{*}{ Ges7 } & F: AGAGGAGTGTGGGAAGGTCTAC & $(\mathrm{TCTT}) 5$ & 56 & 247 \\
\hline & R: GCTAGTTTGGGACGTACTTTAA & & & \\
\hline \multirow[t]{2}{*}{ Gcs8 } & F: GCAGTAGTGTGTCTTGTGTGAG & $($ GGCAGA)5 & 61 & 216 \\
\hline & R: GGGGCTTCAATTCAGGGTTTCC & & & \\
\hline \multirow[t]{2}{*}{ Ges 9} & F: GGGTTCGATCGTCACAAGTTCA & $(\mathrm{AG}) 7(\mathrm{TG}) 9$ & 62 & 153 \\
\hline & R: TCTTCTCTCTCGCGCCCAATTC & & & \\
\hline \multirow[t]{2}{*}{ Gcs 10} & F: CCCGGGTGACAAGGCTTTT & $($ GAAACA) 5 & 57 & 243 \\
\hline & R: TGGAAATGTTAAGGTCACCGAC & & & \\
\hline \multirow[t]{2}{*}{ Gcs11 } & F:TGGCTAAAAGGGACACAAAAGA & (G) $10 \mathrm{n}(\mathrm{G}) 10$ & 58 & 198 \\
\hline & R: TTTTCGTAGGCAGAGAGAATGG & & & \\
\hline \multirow[t]{2}{*}{ Gcs12 } & F: TCCCCATCCTCATCACCATCGT & (GTC)5(TCA)7 & 62 & 156 \\
\hline & R: AAGATTGCCCTCCTCCTCCTGA & & & \\
\hline
\end{tabular}

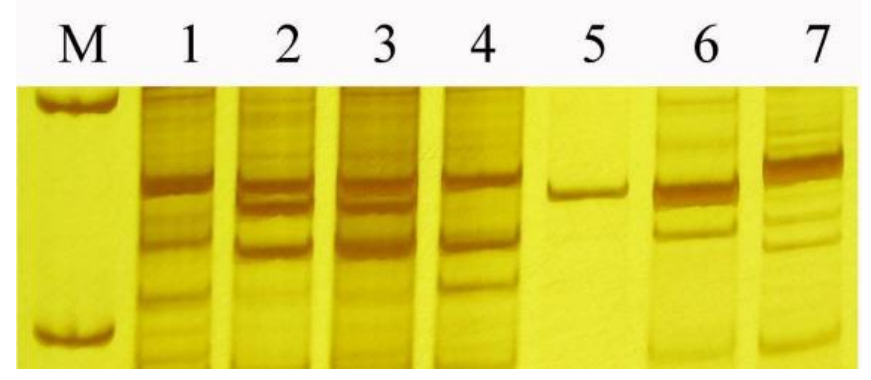

Figure 2 PCR of the primers Gcs6 in 7 Glycyrrhiza uralensis Fisch.

Note: M: D2000 DNA Marker; 1 7: Represent the number of Glycyrrhiza uralensis Fisch. material (Table 1)

\section{Discussion}

In recent years, with the continuous development of sequencing technology, more and more plant genome sequences such as Salvia miltiorrhiza, Dendrobium catenatum, Chrysanthemum, Mentha longifolia and Glycyrrhiza uralensis Fisch. have been published (Song et al., 2018). These data laid the foundation for the development of molecular markers based on sequencing technology, such as SSR (Song et al., 2019), EST-SSR, SNP (Yang et al., 2019), Indel (Liu et al., 2019) and KASP, and their application to identification of germplasm 
resources. In this study, 193207 SSR loci were screened out using MISA software based on the whole genome sequences in Glycyrrhiza uralensis Fisch.. The genome has an average of 482 SSR loci per Mb sequence. The study found that using MISA software to screen SSR loci based on the whole genome, 117511 SSR loci were screened out from $239 \mathrm{Mb}$ genome sequences of Apocynum venetum, with an average of $492 \mathrm{SSR}$ loci $\mathrm{Mb}^{-1} .488$ 592 SSR loci were screened out from $1.18 \mathrm{~Gb}$ genome sequences of Eucommia ulmoides, with an average of 404 SSR loci $\mathrm{Mb}^{-1}$ (Wu et al., 2015). The Corchorus capsularis genome was about $338 \mathrm{Mb}$, with an average of 467 SSR loci $\mathrm{Mb}^{-1}$ (Yang et al., 2019). The genome size of tetraploid Arachis hypogaea Linn., which belongs to legume like Glycyrrhiza uralensis Fisch., was about $2.7 \mathrm{~Gb}$, with an average of $245 \mathrm{SSR} \mathrm{Mb}^{-1}$ (Wang et al., 2019). The Glycyrrhiza uralensis Fisch. genome was about $400 \mathrm{Mb}$, and the distribution frequency of SSR loci in the whole genome was similar to these species, basically in line with the tendency that genome size was negatively correlated with the number or distribution frequency of SSR (Morgante et al., 2002).

It was found that in most species genomes, the distribution frequency of genome SSRs will gradually decrease with the increase of the number of repeat units. Dinucleotide and trinucleotide repeats were the main repeat types, and the difference lies in the difference of repeat units (Sonnet et al., 2000). In this study, 1 6 nucleotide loci existed in the whole genome SSR loci of Glycyrrhiza uralensis Fisch., with the largest number of mononucleotide repeat units, accounting for $60.73 \%$, followed by dinucleotide and trinucleotide repeat units, accounting for $26.11 \%$ and $10.95 \%$ respectively, which was consistent with the results that the proportion of mononucleotide repeat units in the genome of Salvia splendens Ker-Gawler (41.6\%) (Wang et al., 2018), Eucommia ulmoides (54.34\%) (Wu et al., 2015) and Punica granatum L. (51.95\%) (Hong et al., 2019) was the highest. And it was similar to the species that the proportion of dinucleotide repeat units was the highest, such as Fagopyrum tataricum (69.72\%) (Fang et al., 2019), Rhododendron simsii Planch. (94.58\%) (Gong et al., 2018), Scutellaria baicalensis Georgi (68.32\%) (Qi et al., 2015), Gastrodia elata B1. (78.88\%) (Zhou et al., 2017). There was a trendency that the distribution frequency of genome SSR loci decreased with the increase of length of repeat units. It was generally believed that the species which has a large number of mononucleotide and dinucleotide repeat units in the genome has a high level of evolution (Toth et al., 2000). The mononucleotide and dinucleotide in the genome of Glycyrrhiza uralensis Fisch. accounted for $86.84 \%$ of all SSR loci, indicating that Glycyrrhiza uralensis Fisch. may have a late origin and a high level of evolution. In this study, the SSR of Glycyrrhiza uralensis Fisch. contained 284 types of abundant repeat units. The number of repeat sequences composed of bases with same motif but different lengths was different. The dominant units were A/T (58.28\%), AG/CT (10.48\%), AT/AT (10.48\%), AC/GT (5.12\%) and AAT/ATT (3.57\%), which was similar to the base preference of Gastrodia elata Bl. (Zhou et al., 2017), Punica granatum L. (Hong et al., 2019) and Eucommia ulmoides (Wu et al., 2015), and $\mathrm{A} / \mathrm{T}$ was the main repeat unit.

There were also many studies on molecular markers of Glycyrrhiza uralensis Fisch., including RAPD markers (Wu et al., 2003), 26 pairs of markers of other legume species (For et al., 2014), 81 pairs of SRAP primers (Ai et al., 2017) and universal primers to amplify ITS and $p s b A-t r n \mathrm{H}$ sequences to achieve the purpose of molecular identification of different primordial Glycyrrhiza uralensis Fisch. (Yang et al., 2017), but generally, the number of markers was very limited. With the development of sequencing technology, both single primer SCoT markers and EST-SSR markers developed by expressing sequence tags (Song et al., 2017; Xin, 2019) have the problem of insufficient marker coverage. In terms of transcriptome data development markers, 10378 SSRs and 7032 SSRs were screened from the transcriptome data of Glycyrrhiza inflata Batalin and Glycyrrhiza glabra L., respectively, and a total of 110 pairs of SSR primers were designed (Liu et al., 2015; Xin, 2019). Obviously, the traditional methods and molecular markers based on sequencing data have been far from meeting the requirements of identification of genetic diversity and genetic relationship of Glycyrrhiza uralensis Fisch.. The number of SSR markers identified in this study reached 701302 pairs, and most of them could amplify target bands in Glycyrrhiza uralensis Fisch. from different sources, indicating that these markers had great application potential and could significantly improve the efficiency of identification, development and molecular marker assisted selection of Glycyrrhiza uralensis Fisch. germplasm resources. 


\section{Materials and Methods}

\subsection{Experimental materials}

The leaves of wild Glycyrrhiza uralensis Fisch. used in the experiment were collected from Shanxi, Ningxia and other places respectively, and dried and preserved with silica gel. While the cultivated licorice was the hydroponic seedlings. Our group collected seeds from the origin and cultured them in hydroponics. The materials included 3 cultivated Glycyrrhiza uralensis Fisch. and 4 wild Glycyrrhiza uralensis Fisch., which were identified by Professor Zhang Shuosheng from Shanxi University of Chinese Medicine (Table 3). Genome DNA was extracted by improved CTAB method (Zhang et al., 2014).

Table 3 Tested samples from different sources

\begin{tabular}{llll}
\hline Code & Location & Growth mode & Phenotype \\
\hline GC1 & Datong City, Shanxi & Wild & Red \\
GC2 & Yuci City, Shanxi & Wild & Red \\
GC3 & Shizuishan City, Ningxia & Wild & Red \\
GC4 & Yanchi County, Xinjiang & Cultivation & Red \\
GC5 & Longxi County, Gansu & Cultivation & Yellow \\
GC6 & Datong City, Shanxi & Cultivation & Red \\
GC7 & Yangqu County, Shanxi & Cultivation & Red \\
\hline
\end{tabular}

\subsection{Extraction of SSR from licorice genome sequence}

We used MISA (https://webblast.ipk-gatersleben.de/misa/) software to search for repeat loci in the published Glycyrrhiza uralensis Fisch. genome (Mochida et al., 2016). The search criteria were as follows: nucleotide (base pair, bp) 1 bp, 2 bp, 3 bp, 4 bp, 5 bp, 6 bp, the repeat times were not less than 10 times, 6 times, 5 times, 5 times, 5 times, 5 times, 5 times successively, and the composite SSR (the distance between 2 SSRs) was less than $100 \mathrm{bp}$.

\subsection{Design of SSR primers}

Primer 3-2.4.0 software was used to extract $300 \mathrm{bp}$ sequence containing SSR repeat locus flanking as template sequence for marker development. The design criteria of primers were as follows: the GC content was $30 \% \sim 70 \%$, the product size was $100 \sim 250 \mathrm{bp}$, the $\mathrm{Tm}$ value was between $55^{\circ} \mathrm{C} \sim 65^{\circ} \mathrm{C}$, the difference of $\mathrm{Tm}$ value between upstream and downstream primers was no more than $5^{\circ} \mathrm{C}$, the primer length was $18 \sim 22 \mathrm{bp}$, and the $\mathrm{GC}$ content of primers was $30 \% \sim 70 \%$.

\subsection{Validation of Glycyrrhiza uralensis Fisch. SSR primers}

The PCR reaction system was $10 \mu \mathrm{L}: 1 \times$ Taq DNA polymerase buffer, $2.5 \mathrm{mmol} / \mathrm{L} \mathrm{MgCl}_{2}, 1 \mathrm{U}$ DNA polymerase, $100 \mu \mathrm{mol} / \mathrm{L}$ dNTPs, $0.2 \mu \mathrm{mol} / \mathrm{L}$ upstream primers, $0.2 \mu \mathrm{mol} / \mathrm{L}$ downstream primers, $50 \mathrm{ng}$ template DNA, and add distilled water to $10 \mu \mathrm{L}$. The PCR reaction conditions were as follows: pre-denaturation at $95^{\circ} \mathrm{C}$ for $4 \mathrm{~min}$, denaturation at $94^{\circ} \mathrm{C}$ for $1 \mathrm{~min}$, renaturation at $55^{\circ} \mathrm{C} \sim 60^{\circ} \mathrm{C}$ for $45 \mathrm{~s}$, extension at $72^{\circ} \mathrm{C}$ for $1 \mathrm{~min}$, and for 35 cycles, and final extension at $72^{\circ} \mathrm{C}$ for $5 \mathrm{~min}$. The amplified products were mixed with $6 \times$ sample solution, and subjected to non-denaturing polyacrylamide gel electrophoresis for $1 \mathrm{~h}$. After the electrophoresis, silver staining was performed.

\section{Authors' contributions}

ZHX was the main writer and reviser of the manuscript; ZSS and ZHX were in charge of the project, mainly responsible for project conception and experimental design; DCH mainly arranged and analyzed the data of the manuscript; WYL and LR gave specific guidance to the experiment; ZD and YMR completed the experiments. All authors read and approved the final manuscript.

\section{Acknowledgments}

This study was jointly funded by Key Research and Development Project in Shanxi Province (201903D221053), Ph.D. Research Startup Fund Project in Shanxi University of Chinese Medicine (2020BK05) and Science and Technology Innovation Team in Shanxi University of Chinese Medicine (2018TD-009). 


\section{References}

Ai P.F., Su S., and Jing Z.Z., 2017, Optimization of SRAP-PCR system on Glycyrrhiza uralensis by orthogonal test design and selection of primers, Hebei Gongye Keji (Hebei Journal of Industrial Science and Technology), 34(1): 7-11

Chinese Pharmacopoeia Commission, ed., 2015, Pharmacopoeia of the People's Republic of China, China Medical Science Press, Beijing, China, pp.86-88

Fang X.M., Huang K.H., Nie J., Zhang Y.L., Zhang Y.K., Li Y.S., Wang W.W., Xu X., Ruan R.W., Yuan X.H., Zhang Z.S., and Yi Z.L., 2019, Genome-wide mining, characterization, and development of microsatellite markers in tartary buckwheat (Fagopyrum tataricum Garetn.), Euphytica, 215 : 183 https://doi.org/10.1007/s10681-019-2502-6

For T.J.A., Erayman M., Ilhan E., Güzel Y., Abdil H., and Eren, 2014, Transferability of SSR markers from distantly related legumes to Glycyrrhiza species, Turkish Journal of Agriculture and Forestry, 38(1): 32-38 https://doi.org/10.3906/tar-1303-47

Gong J.Y., Liu H., Zhang X.M., Sun W., and Li F., 2018, Characteristic analysis of microsatellite sites and primers development in sequences of Rhododendron spp., Fenzi Zhiwu Yuzhong (Molecular Plant Breeding), 16(22): 7403-7406

Hong W.J., Hao Z.X., Liu K.J., Luo H., Bi R.X., Zhao H., Zong S.X., and Wang J., 2019, Development and identification of SSR molecular markers based on whole genomic sequences of Punica granatum, Beijing Linye Daxue Xuebao (Journal of Beijing Forestry University), 41(8): 38-47

Li B.N., Nan B., Liu C.S., Zhou Y.Q., and Liu J., 2010, Analysis on genetic diversity of licorice Glycyrrhiza uralensis from geo-authentic product areas by ISSR markers, Zhongguo Shiyan Fangjixue Zazhi (Chinese Journal of Experimental Traditional Medical Formulae), 16(12): 90-93

Li X.L., Lu J.H., Xie L.B., Zhang A.X., Chen X.C., and Li X.Y., 2015, Development of EST-SSR primers and genetic relationship analysis in four Glycyrrhiza L. species, Xibei Zhiwu Xuebao (Acta Botanica Boreali-Occidentalia Sinica), 35(3): 60-65

Li X.Y., 1993, A study of the system and new taxa of genus Glycyrrhiza L., Zhiwu Yanjiu (Bulletin of Botanical Research), 13(1): 13-43

Liu W., Zhang Q.Q., Shu F., Cai Y.L., Ma X.L., and Bian Y.B., 2019, Genome-wide SNP/Indel analysis and the construction of genetic linkage maps based on Indel markers of Morchella importuna, Junwu Xuebao (Mycosystema), 38(12): 2195-2204

Liu T., Guo J.F., Na R., and Xue D., 2015, The analysis of differential expression of Glycyrrhiza uralensis root gene by nitrogen ions implantation based on cDNA-AFLP method, Henongxue Bao (Journal of Nuclear Agricultural Sciences), 29(6): 1074-1080

Liu Y.L., Zhang P.F., Song M.L., Hou J.L., Qing M., Wang W.Q., and Liu C.S., 2015, Transcriptome analysis and development of SSR molecular markers in Glycyrrhiza uralensis Fisch., Plos One, 10(11): e0143017

https://doi.org/10.1371/journal.pone.0143017 PMid:26571372 PMCid:PMC4646622

Luo L., Zhang D.D., Li W.B., Yang X., Wang W.Q., and Hou J.L., 2018, Comparison on traits and quality characteristics of cultivated Glycyrrhiza uralensis in different areas, Zhongyaocai (Journal of Chinese Medicinal Materials), 41(4): 829-833

Mochida K., Sakurai T., Seki H., and Yoshida T., 2016, Draft genome assembly and annotation of Glycyrrhiza uralensis, a medicinal legume, Plant J., 89(2): 181-194

https://doi.org/10.1111/tpj.13385

PMid:27775193

Morgante M., Hanafey M., and Powell W., 2002, Microsatellites are preferentially associated with nonrepetitive DNA in plant genomes, Nat. Genet., 30(2): 194-200 https://doi.org/10.1038/ng822 PMid:11799393

Qi L.J., Long P., Jiang C., Yuan Y., and Huang L.Q., 2015, Development of microsatellites and genetic diversity analysis of Scutellaria baicalensis Georgi using genomic-SSR markers, Yaoxue Xuebao (Acta Pharmaceutica Sinica), 50(4): 500-505

Song C., Liu Y.F., Song A.P., Dong G.Q., Zhao H.B., Sun W., Ramakrishnan S., Wang Y., Wang S.B., Li T.Z., Niu Y., Jiang J.F., Dong B., Xia Y., Chen S.M., Hu Z.G., Chen F.D., and Chen S.L., 2018, The Chrysanthemum nankingense genome provides insights into the evolution and diversification of Chrysanthemum flowers and medicinal traits, Mol. Plant, 11(12): 1482-1491

https://doi.org/10.1016/j.molp.2018.10.003

PMid:30342096

Song F., Lu J.H., Han C., Niu Q.D., Cheng C.N., and Li X.Y., 2017, Comparison between SCoT and EST-SSR markers in detection of genetic diversity of wild Glycyrrhiza L. species, Shihezi Daxue Xuebao (Journal of Shihezi University (Natural Science)), 35(2): 213-219

Song L.X., Li G.Q., Jin C.Q., and Gong S.P., 2019, Whole genome sequencing and development of SSR markers in Apocynum cannabinum, Zhiwu Yichuan Ziyuan Xuebao (Journal of Plant Genetic Resources), 20(5): 1309-1316

Sonnet H., Carpendale S., and Strothotte T., 2000, Long microsatellite alleles in Drosophila melanogaster have a downward mutation bias and short persistence times, which cause their genome-wide under representation, Genetics, 155(3): 1213-1220 https://doi.org/10.1093/genetics/155.3.1213

PMid:10880482 PMCid:PMC1461152

Toth G., Gáspári Z., and Jurka J., 2000, Microsatellites in different eukaryotic genomes: survery and analysis, Genome Res., 10(7): 967-981

https://doi.org/10.1101/gr.10.7.967

PMid:10899146 PMCid:PMC310925

Wang S., Ge X.X., Kong W.Y., Chen H. W., Liu K.F., and Wang H.L., 2018, Genome survey and characteristic analysis of SSR in Slvia splenden, Beijing Nongxueyuan Xuebao (Journal of Beijing University of Agriculture), 33(2): 15-22 
Wang Y.L., Huang B.Y., Wang S.Y., Du P., Qi F.Y., Fang Y.J., Sun Z.Q., Zheng Z., Dong W.Z., and Zhang X.Y., 2019, Development and characterization of whole genome SSR in tetraploid wild peanut (Arachis monticola), Zhongguo Nongye Kexue (Scientia Agricultura Sinica), 52(15): 2567-2580

Wu M., Du H.Y., Wu Y.T.N., Li P.F., and Jing T., 2015, Characterization of genomic microsatellites and development of SSR markers of Eucommia ulmoides, Linye Kexue Yanjiu (Forest Research), 28(3): 387-393

Wu X., Liu Q.H., Ma Y.H., and Dang R.L., 2003, RAPD analysis of genetic relationship among different populations of Glycyrrhiza uralensis Fisch .ex DC from six growing area in Sinkiang, Zhongguo Shenghua Yaowu Zazhi (Chinese Journal of Biochemical Pharmaceutics), 24(4): 191-193

Xin Q., 2019, Hybridization area composition and interspecies introgression Glycyrrhiza L. revealed by transcriptome microsatellite markers, Thesis for M.S., Shihezi University, Supervisor: Lu J.H., pp.8-10

Yang B., Cheng M.J., Du Q.X., Zhu J.L., Du H.Y., and Yang S.B., 2019, SNP sites developed by whole genome resequencing analysis in Eucommia ulmoides 'Hongye'. Zhuwu Yanjiu (Bulletin of Botanical Research), 39(6): 947-954

Yang R., Li W.D., Ma Y.S., Zhou S., Xue Y.T., Lin R.C., and Li C., 2017, The molecular identification of licorice species and the quality evaluation of licorice slices, Yaoxue Xuebao (Acta Pharmaceutica Sinica), 52(2): 318-326

Zhang X.Y., Zhao C.Y., Hou H.S., and Tong S.M., 2014, Comparison of DNA extraction methods from four Chinese traditional medical herbs, Zhongguo Shengwu Yaowu Zazhi (Chinese Journal of Biochemical and Pharmaceuticals), 35(7): 17-21

Zhou T.H., Ding J.X., Tian W., and Wang J., 2017, Genomic microsatellite characteristic analysis and molecular marker development for Gastrodia elata BI, Xibei Zhiwu Xuebao (Acta Botanica Boreali-Occidentalia Sinica), 37(9): 1728-1735 